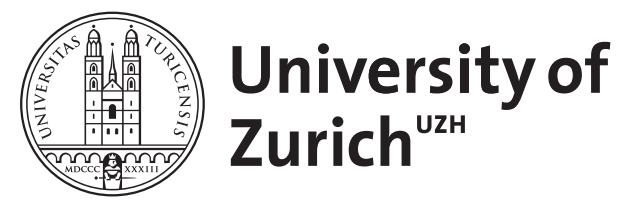

\title{
Osteomyelitis and arthritis
}

\author{
Stumpe, K D M ; Strobel, K
}

\begin{abstract}
Infections of bone and the joints can represent major diagnostic and therapeutic challenges to all clinicians. Together with osteomyelitis and septic arthritis, soft-tissue infections like cellulites/fasciitis and abscess formation can occur, which have to be treated appropriately. Bone scintigraphy is a sensitive method that can be used to search for bone and joint infections. Labeled leukocytes often are used as the gold standard to identify infectious foci in the musculoskeletal system, but major drawbacks of this method are the imaging of chronic infections and imaging of the axial skeleton. Like (111)In-labeled leukocyte imaging, $(99 \mathrm{~m})$ Tc-labeled antigranulocyte antibody scintigraphy has a role in the imaging of osteomyelitis of the peripheral skeleton. Magnetic resonance imaging is widely used to evaluate musculoskeletal infections and is excellent in identifying abscess formation, but the extent and spread of infection is sometimes difficult to delineate because hyperemia and infection are not congruent.
\end{abstract}

DOI: https://doi.org/10.1053/j.semnuclmed.2008.08.003

Posted at the Zurich Open Repository and Archive, University of Zurich

ZORA URL: https://doi.org/10.5167/uzh-32343

Journal Article

Accepted Version

Originally published at:

Stumpe, K D M; Strobel, K (2009). Osteomyelitis and arthritis. Seminars in Nuclear Medicine, 39(1):2735.

DOI: https://doi.org/10.1053/j.semnuclmed.2008.08.003 


\section{Osteomyelitis and Arthritis}

Katrin D.M. Stumpe, MD and Klaus Strobel, MD

Division of Nuclear Medicine, Department of Medical Radiology, University Hospital, Zurich, Switzerland

Address of correspondence: Katrin D.M. Stumpe, MD

Division of Nuclear Medicine

Department of Medical Radiology

University Hospital

8091 Zurich

Switzerland

Phone: ++41 442553479

Fax: ++41 442554414

katrin.stumpe@usz.ch 


\begin{abstract}
Infections of bone and joints can represent major diagnostic and therapeutic challenges to all clinicians. Together with osteomyelitis and septic arthritis, soft tissue infections like cellulites/fasciitis and abscess formation can occur, which have to be treated appropriately. Bone scintigraphy is a sensitive method to look for bone and joint infections. Labeled leucocytes are aften used as gold standard to identify infectious foci in the musculoskeletal system, but major drawbacks of this method are the imaging of chronic infections and imaging of the axial skeleton. Like 111 In - labeled leucocyte imaging, 99m Tc - labeled antigranulocyte antibody scintigraphy has a role in imaging of osteomyelitis of the peripheral skeleton

Magnetic resonance imaging (MRI) is widely used to evaluate musculoskeletal infections and is excellent in identifying abcess formation, but the extent and spread of infection is sometimes difficult to delineate because hyperemia and infection are not congruent.
\end{abstract}

Recent studies indicate that 18 F-2-fluoro-2-deoxy-D-glucose (FDG) positron emission tomography (PET) has considerable value for diagnosing inflammatory and infectious disease of the axial and peripheral skeleton. FDG PET makes use of the fact that there is no physiological FDG accumulation in white cells not actively fighting an infection which permits excellent imaging of the axial skeleton, especially in patients with infection of the spine. PET/CT seems to be more accurate for confirming or excluding low-grade infection and chronic osteomyelitis than 99m Tc bone scintigraphy, 99m Tc - labeled antigranulocyte antibody scintigraphy and $111 \mathrm{In}$ labeled leucocyte scintigraphy. While this usefulness extends to infections associated with metallic implants used for trauma surgery, PET may not be as useful in the diagnosis of infections associated with prosthetic joints. Compared to PET alone, PET/CT offers additional 
information as it provides precise anatomical information and characterisation of the infectious lesion which is important for surgical planning.

\section{Osteomyelitis}

Choosing the appropiate combination of imaging methods in the evaluation of infection may be challenging. A variety of methods are available for imaging inflammation and infection. The role of imaging is to confirm the clinical suspicion, characterize the lesion and its extent and detect complications such as abscess or fistula formation in infectious disease.

Standard radiography and magnetic resonance imaging (MRI) are commonly used to detect inflammatory and infectious lesions in the bone. Radiographs should always been performed to get an anatomic overview of the region of interest and to select subsequent imaging modalities. MRI has been widely used because of its excellent soft tissue contrast and its sensitivity to tissue edema and hyperemia. It is especially valuable for septic arthritis, spinal infection and diabetic foot infections. However, these modalities are of limited value to detect early infection when morphological changes are still missing. In addition, diagnostic difficulties always arise when chronic infection is suspected particularly when there are preexisting alterations in the spine due to trauma, surgery, or infection. Artifacts caused by prosthetic joints or metallic implants in the spine or extremities can degrade images sufficiently to make diagnosis impossible in both CT or MRI. 
Therefore nuclear medicine procedures are needed as a functional adjunct to complement morphologic imaging techniqes.

The choice of the best nuclear medicine procedure depends on the grade of inflammation, duration of infection, availability, cost, and radiation exposure. Mainly used radiopharmaceuticals of conventional nuclear medicine comprise:

Three-phase bone scintigraphy, 67 Ga-citrate, 111 In- and 99m Tc-HMPAO-labeled leucocytes, 99m Tc-radiolabeled murine monoclonal antigranulocyte antibodies (MAB) and 99m Tcradiolabeled nanocolloids and human immunoglobulins (HIG).

Three-phase bone scintigraphy is readily available and accurate in unviolated bone. However it is non-specific in patients with previously violated bone, in patients with prosthetic joints and in the neuropathic joint. Under these circumstances sequential bone/gallium 67 scintigraphy is used, however, specificities still vary between less than 50\% and 100\% (1-4). However the need to perform two imaging techniques and delayed imaging are major disadvantaged. Gallium-67 imaging alone is useful as an adjunct to MRI in spinal infection.

Labeled leucocytes and antigranulocyte antibodies are neither sensitive nor specific for infection in the axial skeleton (5-7). The latter imaging techniques have appropriate diagnostic accuracy in the peripheral skeleton, however differentiation between soft tissue and bone infection is often impossible due to limited spatial resolution (Fig. 1).

Among the various conventional nuclear medicine procedures, 111-Indium labeled leucocytes is one of the most specific imaging technique and useful in acute infection, in osteomyelitis of the diabetic foot and in the neuropathic joint. In addition, labeled leucocytes together with complementary bone marrow scintigraphy is the radionuclide procedure of choice in prosthetic 
joint infection. However, presentation of patients with prosthetic joints and the diabetic foot is complex and discussed in separate articles in this edition on infectious disease.

The development of integrated computed tomography (SPECT)-computed tomography (CT) cameras has overcome partly the lack of spatial resolution in conventional nuclear medicine by using image coregistration (8).

Fluorine-18 (F-18) fluorodeoxyglucose-positron emission tomography (FDG PET) has shown some advantages in constrast to other imaging methods, and this is due to the so-called ‘respiratory burst' which mononuclear cells and neutrophilic granulocytes undergo when activated and while fighting an infection $(9,10)$. Infection can be acute or chronic, the former showing predominantly neutrophilic granulocytes infiltrates, whereas in the latter macrophages join the neutrophils.

In contrast to acute osteomyelitis, low grade and chronic infections are more difficult to diagnose with the current imaging modalities. In this setting, PET is successfully performed because FDG is avidly taken up by activated macrophages in the chronic phases of infection. FDG PET has the highest diagnostic accuracy for confirming or excluding chronic osteomyelitis (11). According to the literature $(12,13)$, a negative FDG PET scan can virtually rule out ostemyelitis.

FDG PET is superior to labeled leucocytes imaging for the detection of chronic osteomyelitis in the axial skeleton as physiologic FDG uptake in the hematopoietic marrow is relatively low (11). According to a recent meta-analysis by Termatt et al. (11) FDG PET is not only the most sensitive examination for the detection of chronic osteomyelitis but also more specific than labeled leucocytes, bone scintigraphy, or MRI in this setting. Due to high physiological uptake in the hematopoetic bone marrow in labeled leucocyte imaging, sensitivities are as low as $21 \%$ to detect chronic osteomyelitis in the axial skeleton (11). 
Increased FDG accumulation in PET is also associated with inflammatory arthritis, fractures, normally healing bone and degenerative changes (14-16). However in contrast to bone scintigraphy, FDG PET rapidly normalizes after traumatic or surgical fractures $(14,16)$ as fibroblast predominate in normally healing bone, and FDG uptake quickly subsides 4 months after surgery (17). The healing process shows most of the cells that are present in inflammation (18). Therefore, specificity is increasing, if recently (less than 4 months) traumatized or operated bone is excluded.

Our group reported on FDG PET in 18 patients with suspected acute and subacute osteomyelitis in the axial and peripheral skeleton and found sensitivities of $100 \%$ and specificities in the range of $83 \%$ to $99 \%$, respectively (19). De Winter et al. (13) prospectively studied FDG PET in 60 patients with chronic osteomyelitis and found sensitivity, specificity and accuracy of $100 \%$, $86 \%$, and 93\%, respectively. In a retrospective study, Kaelicke et al. (20) reported on 15 true positive findings in 15 patients with histologically confirmed acute $(n=7)$ and chronic osteomyelitis $(n=8)$.

FDG PET was superior to $99 \mathrm{~m}$ Tc-labeled antigranulocyte antibody scintigraphy in the evaluation of chronic osteomyelitis involving in the axial skeleton ( $\mathrm{n}=15$ out of 51) (12). 99m Tc-labeled antigranulocyte antibody scintigraphy frequently cannot differentiate between active and inactive processes due to nonspecific areas of decreased radionuclide uptake. FDG PET provided sufficient anatomical information and spatial resolution to distinguish soft tissue from bone infection, despite the presence of metallic implants. In another study by Guhlmann et al. (21) overall sensitivity and specificity were $100 \%$ and $92 \%$, respectively, in the assessment of chronic osteomyeltitis in the peripheral $(n=21)$ and axial skeleton $(n=10)$. In the latter study, FDG PET showed particulary promising results in the detection of chronic osteomyelitis in the 
axial skeleton, an area where labeled white cell scanning is of limited value with an accuracy in the range between $53 \%$ and $76 \%(22)$.

In a study performed by Zhuang et al. (23) sensitivity, specificity and accuracy were $100 \%$, $87.5 \%$ and $90.9 \%$ for FDG PET in 22 patients with suspected chronic osteomyelitis. Chacko et al. (24) reviewed the results of 167 FDG PET scans in patients with the suspicion of various infections. Fifty-six patients out of these 167 patients were suspected to have chronic osteomyelitis with an accuracy of $91 \%$. In addition, the available data show that FDG PET is superior to conventional nuclear medicine methods to distinguish between soft tissue and bone infection (Fig. 2).

FDG PET and FDG PET/CT have many advantages over conventional medicine imaging techniques: completion within one hour, high sensitivity, high target to background contrast and high-resolution tomographic images. PET/CT with the combination of PET and a low-dose or full-dose diagnostic CT helps in this setting as it provides exact anatomic localisation of FDG uptake and increases the specificity compared to PET alone. The logistics of the PET technique make its use easier in chronic than in acute inflammation.

\section{Disc space infection:}

The diagnosis of disc space infection has always been a challenge for the clinician. Conventional radiography is normal within the first 8 weeks before structural changes occur. The diagnostic imaging technique of choice is contrast enhanced MRI with an accuracy of approximately $90 \%$ which provides early diagnosis of disc space infection $(25,26)$. In addition to bone marrow abnormalities, epidural, subdural, intramedullary and paraspinal soft tissue changes are signs for 
spinal infection which can be clearly delineated in contrast enhanced MRI. However, differentiation between degenerative (so-called Modic abnormalities) and infectious disc disease can be difficult due to potentially similar abnormalities.

Three-phase bone scintigraphy is of limited value in the differentiation of degenerative from infectious end plate abnormalities. It showed an overall accuracy of only $67 \%$ and cannot be recommended in the clinical routine (27). Modic et al. (25) described a sensitivity and specificity of $90 \%$ and $78 \%$ for bone scintigraphy and $96 \%$ and $92 \%$ for MRI, respectively. Bone scintigraphy together with gallium 67 SPECT is the radionuclide imaging method of choice so far for diagnosing spinal osteomyelitis. Love et al. (4) reported that gallium 67 scintigraphy was more sensitive than bone scintigraphy and, when performed as SPECT was comparable to sequential bone-gallium scintigraphy with sensitivities of $91 \%$ and specificities of $77 \%$, respectively. In contrast to bone scintigraphy, Gallium 67 scintigraphy can more easily identify local extension of spinal infection (e.g. soft tissue infection). In addition, Gallium 67 is more useful in monitoring treatment response as it more accurately reflects the degree of activity of infective processes (28). 111-Indium labeled leucocytes have a limited value in the diagnosis of spinal infection due to photopenic defects, which are unspecific as several non-infectious conditions (e.g. tumors and infarction) tend to show photopenia $(29,30)$. Although most of the published series are small, FDG PET appears to be superior in the evaluation of spinal osteomyelitis with higher sensitivities and specificities compared to gallium 67-citrate imaging (31-33). In a study performed by Gratz et al. (33) using a coincidence camera, FDG PET was superior to MRI and gallium imaging in patients with suspected disc space infection. Schmitz et al. (32) reported that FDG PET is sensitive in the detection of disc space infection and additional paravertebral soft tissue involvement. Sixteen out of 12 patients had a histopathologically 
confirmed disc space infection. According to our results, FDG PET appears to be useful for excluding disc space infection in equivocal MR cases. In the latter PET study, we included 30 symptomatic patients with substantial end plate abnormalities of the lumbar spine in MR imaging (31). FDG PET did not show uptake in the intervertebral spaces of any patient with degenerative disease, whereas infectious end plate abnormalities were always positive (Fig. 3). The sensitivity and specificity for MRI in detecting disc space infection were $50 \%$ and $96 \%$, and for FDG PET 100\% and 100\%, respectively.

\section{Metallic implant associated infections in trauma patients (except prosthetic joints):}

In traumatology, standard radiography may demonstrate non-union, sequestered bone, intraosseous abscess formation and bone resorption at implant-bone interfaces in infection. Standard radiography is not useful, however, for the diagnosis of early or low-grade infection. MR imaging demonstrates abnormalities of bone marrow and soft tissue if not hampered by susceptibility artifacts caused by metallic implants. However, reparative and infected tissue may be impossible to differentiate. This is also true for ultrasound which is also limited to bone surfaces and soft tissue. CT more precisely demonstrates fragments and sequesters than standard radiography but is inferior to MR imaging in soft tissue and bone marrow assessment. In conventional nuclear medicine, three-phase bone scintigraphy is used for the initial evaluation for osteomyelitis, but findings are affected by previous surgery and trauma and are often not specific. The limitations of spatial resolution are a relevant problem. Specificity increases if combined bone and gallium scanning is used.

In the last decade $\left[{ }^{111} \mathrm{In}\right]$ labeled leukocytes combined with ${ }^{99 \mathrm{~m}} \mathrm{Tc}$ bone marrow scintigraphy have been shown to be highly accurate for the diagnosis of various musculoskeletal 
infection which could alter distribution of bone marrow with sensitivities and specifities of $100 \%$ and $94 \%$, respectively (34).

Labeled leucocyte scintigraphy combined with bone marrow scintigraphy is the conventional radionuclide procedure of choice for diagnosing complicating osteomyelitis like in trauma patients with metallic implants. FDG PET represents a promising imaging technique in the diagnosis of implant-associated infections in trauma patients imaging and has shown to be both sensitive and specific $(13,35)$. Conventional radionuclide methods are often first-line imaging procedures in the diagnosis of implant-associated infections in patients with trauma so far. However, nonspecific tissue uptake of imaging agents and imaging over several days restrict their usefulness. Unlike MRI and CT, FDG PET images are not substantially affected by metallic implants inserted for fixing fractures in contradistinction to PET imaging in prosthetic joint devices. This is most likely due to the more slender materials (e.g. titanium) and methods (e.g. external fixation) used in trauma patients (Fig. 4 and 5). Patients with prosthetic joint devices show artifacts due to relatively high photon absorption of the prosthesis $(35,36)$. FDG PET demonstrates a sensitivity of nearly $100 \%$ and a specificity in the range of $88 \%$ to $93 \%$ in the diagnosis of chronic musculoskeletal infections, including patients with and without metallic implants or prosthetic replacements $(13,21,35)$. De Winter et al. (13) evaluated the use of FDG PET in the diagnosis of chronic musculoskeletal infections in 34 patients with metallic implants. Seventeen patients demonstrated infections around metallic implants used in trauma surgery. Infectious lesions in the peripheral, as well as in the axial skeleton were correctly identified with FDG PET. This in contrast to the results of 17 patients with suspected periprosthetic infections. Similar results were found by Guhlmann et al. (21), who examined six patients with suspected 
metallic implant-associated infection of a group of 31 patients with suspected chronic osteomyelitis. The only false-positive finding was a patient with a soft-tissue infection, which in PET was localized to bone because of missing anatomic landmarks. In a study performed by Kälicke et al. (20) FDG PET was true positive in all cases. FDG PET was not affected by metallike implants used for fixation of fractures. Moreover, Kälicke et al. (20) demonstrated that FDG uptake at the sites of fractures and non-unions is significantly lower than it is at the sites of infections, thereby facilitating differentiation. Results of our own data showed a sensitivity of $100 \%$, a specificity of $93 \%$ and an accuracy of $97 \%$ with FDG PET in the diagnosis of metallic implant-associated chronic infections in 22 patients (29 scans) with a prior history of trauma (35). One false-positive finding was detected in the soft tissue of a patient six weeks after surgery and no false-negative findings were seen. In addition, the surgeons assessed the influence of FDG PET on their treatment decisions. FDG PET influenced the clinical decisionmaking process in almost two thirds of the patients. FDG PET could accurately differentiate between bone and soft tissue infection.

One of the largest studies was prospectively performed by de Winter et al. (37). The latter group investigated FDG PET in 57 patients ( $\mathrm{n}=27$ with metallic implants) with suspected infection after prior spinal surgery. The median interval between surgery and FDG PET examination was 10 months (range 1.25-288 months). Infection was detected in 10 of 27 patients with, and in 5 of 30 patients without metallic instrumentation in the spine. Sensitivity, specificity and accuracy was $100 \%, 81 \%$ and $86 \%$, respectively. In about $60 \%$ of patients, infection could be ruled out with FDG PET. Although specificity of FDG PET was not higher than $81 \%$, comparable results are not obtained with bone scintigraphy, labeled leucocyte or MRI in the postoperative spine (38). 
In a recent study performed by our own group, we evaluated the diagnostic value of FDG PET/CT in trauma patients with suspected chronic osteomyelitis and found promising results (Fig. 6) (39). Of 33 PET/CT scans, 17 were true positive, 13 true negative, 2 false positive and 1 false negative. Sensitivity, specificity and accuracy for FDG PET/CT was 94\%, 87\% and 91\% for the whole group, $88 \%, 100 \%$ and $90 \%$ for the axial skeleton and $100 \%, 85 \%$ and $91 \%$ for the peripheral skeleton, respectively. Our data showed two false positive (both in the peripheral skeleton) and one false negative finding (in the axial skeleton). Summarizing the results of these studies, FDG PET is superior for detecting chronic osteomyelitis in the axial skeleton in contrast to labeled leucocyte imaging (11).

PET/CT allowed precise localization of the infectious focus and demonstrated the extent of chronic osteomyelitis with a high degree of accuracy.

\section{Conclusion}

Bone scintigraphy, gallium-67, and labeled leucocytes are the conventional nuclear medicine imaging techniques of choice for imaging musculoskeletal infection.

At the present time, FDG PET has an incremental value over other imaging modalities and seems to be more sensitive and specific in the detection of various infectious diseases. In the diagnosis of osseous infection, FDG PET has a major impact in patients with chronic osteomyelitis. Particulary in the axial skeleton, FDG PET is an important imaging technique in the diagnosis and exclusion of chronic osteomyelitis, showing superior accuracy to other radionuclide imaging modalities. Moreover FDG PET plays an important role in the differentiation between disc space infection and erosive degenerative disc disease, where both MRI and bone scan may be falsely positive. FDG PET may replace other imaging modalities in the assessment of metallic implant 
associated infection in trauma patients (excluding prosthetic joints). Differentiation between osteomyelitis and soft tissue infection may be better obtained with FDG PET than with CT or MRI, because of better lesion-to-background contrast and hardly no artifacts arising from metallic implants used in trauma surgery compared with CT and MRI. FDG PET/CT permits more precise delineation and characterization of the infectious focus and helps to improve the management of patients with various infectious diseases.

\section{Septic arthritis}

Bacterial arthritis may be caused by penetrating trauma or through ulcers such as those occurring in diabetes. Direct implantation of infectious agents may also occur after injections or surgery. Hematogeneous routes of infection occur especially in immunocompromised patients. While bone scintigraphy is widely used in imaging non-infectious arthritis in rheumatologic patients, radionuclide imaging has a limited role in the management of septic arthritis because ultrasound and joint fluid aspiration with microbiologic work-up are easy to perform and everywhere available. Radionuclide imaging can be useful in the differentiation of septic arthritis from osteomyelitis and soft tissue infection as well as in the detection of multifocal joint infections.

The appearance of septic arthritis in three-phase bone scintigraphy with $99 \mathrm{~m} \mathrm{Tc}$ - diphosphonates is characteristic: due to hyperaemia in synovial vessels all three phases (the perfusion-, blood pool- and osseous phase) show increased radionuclide uptake (40-42). Normal or even decreased radiotracer uptake can occur in cases of arthritis if the blood flow is compromised by high intracapsular pressure. Majd et al. found increased uptake in six of 7 children and decreased uptake in one of 7 children with proven septic arthritis (43). Bone scintigraphy is more sensitive than standard radiography in the diagnosis of arthritis because radionuclide uptake precedes 
morphological bone changes $(41,44)$. If the bone scan is negative but the clinical impression is still suspicious for septic arthritis specificity of bone scans can be improved by the additional performance of Ga-67-, labeled leucocytes- or monoclonal antibody studies (Fig. 6). However, the need to use multiple radionuclide tracers and to perform imaging at multiple times adds complexity, delays start of treatment and causes inconvenience to the patients. SPECT and SPECT/(CT) are useful supplementations of planar scintigraphy especially in equivocal cases and in the differentiation of soft tissue and bone infection as demonstrated in a patient with osteomyelitis $(8,45)$. SPECT/(CT) should also be considered in patients with arthritis in complex body regions like the spine $(46,47)$.

MRI with intravenous contrast administration reliably demonstrates the abnormalities associated with septic arthritis, including joint effusion, synovial hyperemia and proliferation. In addition, reactive bone marrow and soft tissue edema, secondary osteomyelitis, tendon abnormalities and soft tissue abscesses are found (48). While MRI might be superior in monoarticular infections, the advantage of bone scintigraphy over MRI is that a bone scan is a whole-body imaging technique and covers all involved joints in one investigation. Thus, in infections involving multiple joints, radionuclide studies should be performed.

The role of FDG PET/CT in the work-up of septic arthritis has not been defined yet. According to our experience, FDG PET/CT is particularly useful in clinically difficult cases where the advantage of PET as a relatively fast, whole-body imaging modality plays an important role as demonstrated in Figure 7. Compared with bone scintigraphy, FDG PET/CT can also discover infectious foci outside the bone like in the lung or other organs. Dumarey et al. showed promising initial results using 18F-FDG labeled leucocytes for infection imaging and reported one case of septic knee arthritis among the 23 patients included (49). Cost effectiveness of 
advanced and expensive imaging modalities like SPECT/CT or PET/CT have not been investigated yet and further studies are needed to define the role of nuclear medicine in septic arthritis compared with morphologic imaging like ultrasound and MRI. 


\section{References}

1. Rosenthall L, Lisbona R, Hernandez M, Hadjipavlou A. 99mTc-PP and 67Ga imaging following insertion of orthopedic devices. Radiology 1979; 133:717-721.

2. Schauwecker DS, Park HM, Mock BH, et al. Evaluation of complicating osteomyelitis with Tc-99m MDP, In-111 granulocytes, and Ga-67 citrate. J Nucl Med 1984; 25:849853.

3. Schauwecker DS. The scintigraphic diagnosis of osteomyelitis. AJR Am J Roentgenol 1992; 158:9-18.

4. Palestro CJ, Torres MA. Radionuclide imaging in orthopedic infections. Semin Nucl Med 1997; 27:334-345.

5. Chung JK, Yeo J, Lee DS, et al. Bone marrow scintigraphy using technetium-99mantigranulocyte antibody in hematologic disorders. J Nucl Med 1996; 37:978-982.

6. Jacobson AF, Gilles CP, Cerqueira MD. Photopenic defects in marrow-containing skeleton on indium-111 leucocyte scintigraphy: prevalence at sites suspected of osteomyelitis and as an incidental finding. Eur J Nucl Med 1992; 19:858-864.

7. Gratz S, Braun HG, Behr TM, et al. Photopenia in chronic vertebral osteomyelitis with technetium-99m-antigranulocyte antibody (BW 250/183). J Nucl Med 1997; 38:211-216.

8. Horger M, Eschmann SM, Pfannenberg C, et al. The value of SPET/CT in chronic osteomyelitis. Eur J Nucl Med Mol Imaging 2003; 30:1665-1673.

9. Babior BM. The respiratory burst of phagocytes. J Clin Invest 1984; 73:599-601.

10. Kaim AH, Weber B, Kurrer MO, Gottschalk J, Von Schulthess GK, Buck A. Autoradiographic quantification of $18 \mathrm{~F}-\mathrm{FDG}$ uptake in experimental soft-tissue abscesses in rats. Radiology 2002; 223:446-451.

11. Termaat MF, Raijmakers PG, Scholten HJ, Bakker FC, Patka P, Haarman HJ. The accuracy of diagnostic imaging for the assessment of chronic osteomyelitis: a systematic review and meta-analysis. J Bone Joint Surg Am 2005; 87:2464-2471.

12. Guhlmann A, Brecht-Krauss D, Suger G, et al. Fluorine-18-FDG PET and technetium99m antigranulocyte antibody scintigraphy in chronic osteomyelitis. J Nucl Med 1998; 39:2145-2152.

13. de Winter F, van de Wiele C, Vogelaers D, de Smet K, Verdonk R, Dierckx RA. Fluorine-18 fluorodeoxyglucose-position emission tomography: a highly accurate imaging modality for the diagnosis of chronic musculoskeletal infections. J Bone Joint Surg Am 2001; 83-A:651-660.

14. Meyer M, Gast T, Raja S, Hubner K. Increased F-18 FDG accumulation in an acute fracture. Clin Nucl Med 1994; 19:13-14.

15. von Schulthess GK, Meier N, Stumpe KD. Joint accumulations of FDG in whole body PET scans. Nuklearmedizin 2001; 40:193-197.

16. Zhuang H, Sam JW, Chacko TK, et al. Rapid normalization of osseous FDG uptake following traumatic or surgical fractures. Eur J Nucl Med Mol Imaging 2003; 30:10961103.

17. Kaim AH, Gross T, von Schulthess GK. Imaging of chronic posttraumatic osteomyelitis. Eur Radiol 2002; 12:1193-1202.

18. Henry G, Garner WL. Inflammatory mediators in wound healing. Surg Clin North Am 2003; 83:483-507. 
19. Stumpe KD, Dazzi H, Schaffner A, von Schulthess GK. Infection imaging using wholebody FDG-PET. Eur J Nucl Med 2000; 27:822-832.

20. Kalicke T, Schmitz A, Risse JH, et al. Fluorine-18 fluorodeoxyglucose PET in infectious bone diseases: results of histologically confirmed cases. Eur J Nucl Med 2000; 27:524528.

21. Guhlmann A, Brecht-Krauss D, Suger G, et al. Chronic osteomyelitis: detection with FDG PET and correlation with histopathologic findings. Radiology 1998; 206:749-754.

22. Palestro CJ, Kim CK, Swyer AJ, Vallabhajosula S, Goldsmith SJ. Radionuclide diagnosis of vertebral osteomyelitis: indium-111-leukocyte and technetium-99m-methylene diphosphonate bone scintigraphy. J Nucl Med 1991; 32:1861-1865.

23. Zhuang H, Duarte PS, Pourdehand M, Shnier D, Alavi A. Exclusion of chronic osteomyelitis with F-18 fluorodeoxyglucose positron emission tomographic imaging. Clin Nucl Med 2000; 25:281-284.

24. Chacko TK, Zhuang H, Nakhoda KZ, Moussavian B, Alavi A. Applications of fluorodeoxyglucose positron emission tomography in the diagnosis of infection. Nucl Med Commun 2003; 24:615-624.

25. Modic MT, Feiglin DH, Piraino DW, et al. Vertebral osteomyelitis: assessment using MR. Radiology 1985; 157:157-166.

26. Meyers SP, Wiener SN. Diagnosis of hematogenous pyogenic vertebral osteomyelitis by magnetic resonance imaging. Arch Intern Med 1991; 151:683-687.

27. Love C, Patel M, Lonner BS, Tomas MB, Palestro CJ. Diagnosing spinal osteomyelitis: a comparison of bone and Ga-67 scintigraphy and magnetic resonance imaging. Clin Nucl Med 2000; 25:963-977.

28. Tyrrell PN, Cassar-Pullicino VN, McCall IW. Spinal infection. Eur Radiol 1999; 9:10661077.

29. Gemmel F, Dumarey N, Palestro CJ. Radionuclide imaging of spinal infections. Eur J Nucl Med Mol Imaging 2006; 33:1226-1237.

30. Whalen JL, Brown ML, McLeod R, Fitzgerald RH, Jr. Limitations of indium leukocyte imaging for the diagnosis of spine infections. Spine 1991; 16:193-197.

31. Stumpe KD, Zanetti M, Weishaupt D, Hodler J, Boos N, Von Schulthess GK. FDG positron emission tomography for differentiation of degenerative and infectious endplate abnormalities in the lumbar spine detected on MR imaging. AJR Am J Roentgenol 2002; 179:1151-1157.

32. Schmitz A, Risse JH, Grunwald F, Gassel F, Biersack HJ, Schmitt O. Fluorine-18 fluorodeoxyglucose positron emission tomography findings in spondylodiscitis: preliminary results. Eur Spine J 2001; 10:534-539.

33. Gratz S, Dorner J, Fischer U, et al. 18F-FDG hybrid PET in patients with suspected spondylitis. Eur J Nucl Med Mol Imaging 2002; 29:516-524.

34. Palestro CJ, Roumanas P, Swyer AJ, Kim CK, Goldsmith SJ. Diagnosis of musculoskeletal infection using combined In-111 labeled leukocyte and Tc-99m SC marrow imaging. Clin Nucl Med 1992; 17:269-273.

35. Schiesser M, Stumpe KD, Trentz O, Kossmann T, Von Schulthess GK. Detection of metallic implant-associated infections with FDG PET in patients with trauma: correlation with microbiologic results. Radiology 2003; 226:391-398. 
36. Stumpe KD, Notzli HP, Zanetti M, et al. FDG PET for differentiation of infection and aseptic loosening in total hip replacements: comparison with conventional radiography and three-phase bone scintigraphy. Radiology 2004; 231:333-341.

37. De Winter F, Gemmel F, Van De Wiele C, Poffijn B, Uyttendaele D, Dierckx R. 18Fluorine fluorodeoxyglucose positron emission tomography for the diagnosis of infection in the postoperative spine. Spine 2003; 28:1314-1319.

38. Rothman SL. The diagnosis of infections of the spine by modern imaging techniques. Orthop Clin North Am 1996; 27:15-31.

39. Hartmann A, Eid K, Dora C, Trentz O, von Schulthess GK, Stumpe KD. Diagnostic value of (18)F-FDG PET/CT in trauma patients with suspected chronic osteomyelitis. Eur J Nucl Med Mol Imaging 2006.

40. Rosenthall L, Kloiber R, Damtew B, Al-Majid H. Sequential use of radiophosphate and radiogallium imaging in the differential diagnosis of bone, joint and soft tissue infection: quantitative analysis. Diagn Imaging 1982; 51:249-258.

41. Gilday DL, Paul DJ, Paterson J. Diagnosis of osteomyelitis in children by combined blood pool and bone imaging. Radiology 1975; 117:331-335.

42. Maurer AH, Chen DC, Camargo EE, Wong DF, Wagner HN, Jr., Alderson PO. Utility of three-phase skeletal scintigraphy in suspected osteomyelitis: concise communication. $\mathrm{J}$ Nucl Med 1981; 22:941-949.

43. Majd M, Frankel RS. Radionclide imaging in skeletal inflammatory and ischemic disease in children. AJR Am J Roentgenol 1976; 126:832-841.

44. Gilday DL. Problems in the scintigraphic detection of osteomyelitis. Radiology 1980; 135:791.

45. Filippi L, Schillaci O. Usefulness of hybrid SPECT/CT in 99mTc-HMPAO-labeled leukocyte scintigraphy for bone and joint infections. J Nucl Med 2006; 47:1908-1913.

46. Michel-Batot C, Dintinger H, Blum A, et al. A particular form of septic arthritis: septic arthritis of facet joint. Joint Bone Spine 2008; 75:78-83.

47. Swayne LC, Dorsky S, Caruana V, Kaplan IL. Septic arthritis of a lumbar facet joint: detection with bone SPECT imaging. J Nucl Med 1989; 30:1408-1411.

48. Learch TJ, Farooki S. Magnetic resonance imaging of septic arthritis. Clin Imaging 2000; 24:236-242.

49. Dumarey N, Egrise D, Blocklet D, et al. Imaging infection with 18F-FDG-labeled leukocyte PET/CT: initial experience in 21 patients. J Nucl Med 2006; 47:625-632. 


\section{Figure captions:}

Figure 1. 72-year old man with peripheral arterial occlusive disease after amputation of the first toe of the foot and with suspicion for osteomyelitis of the second toe of the right foot.

99m-Tc labeled antigranulocyte antibody scintigraphy shows focally increased radionuclide uptake of the distal tip of the second toe after $5 \mathrm{~h}$ (a) and $24 \mathrm{~h}$ (b ventral view,c right lateral view). Differentiation between soft tissue or bone infection was not possible. After partial amputation of the second toe of the right foot, histopathology confirmed osteomyelitis.

Figure 2. 75-year-old man with adenocarcinoma of the left upper lobe. In addition the patient presented with an abscess in the right thoracic wall. Maximum-intensity-projection MIP (a) demonstrating circular increased FDG uptake in the left upper lung (short arrow) as well as diffuse FDG uptake in the right upper hemithorax (long arrow). Physiological FDG uptake is seen in the scaleni muscles as well as in the larynx. Axial CT scan (b) demonstrates the lung tumor in the right upper lobe (short arrow) and a tumorous lesion subpectoral (arrowheads) in the right thoracic wall with air and involvement of the right sterno-clavicular joint (long arrow). Axial PET/CT scan (c) shows increased FDG uptake in the bronchial carcinoma in the left upper lobe. In addition circular increased FDG uptake is seen in the tumorous lesion in the right hemithorax representing the abscess as well as focally increased FDG uptake of the right sternoclavicular joint and the adjacent bones with osseous destruction. In addition the right sternocostal joint was involved. In contrast to PET or CT imaging alone, co-registrated PET/CT helps to diagnose soft tissue infection and additional infectious involvement of the adjacent bones.

Figure 3. 72-year-old man with fever of unknown origin. Maximum-intensity-projection MIP (a) 
showing linear increased FDG uptake in the upper and lower lumbar spine (black arrowheads). Sagittal PET/CT image shows FDG uptake in the endplates of the vertebral bodies L1/2 and L4/5 (a, white arrowheads). On axial PET/CT scan (b), additional increased FDG accumulation in the soft tissues ventrally to L1/2 is seen (c). Disc space infection at L1/2 and L4/5 intervertebral disc level was confirmed .

Figure 4. 19-year-old woman with pain and increased inflammatory blood parameters 18 months after lumbar spine stabilisation surgery because of severe scoliosis. Maximum-intensity projection MIP (a) showing increased FDG accumulation in the lower thoracic and upper lumbar spine (black arrow). Axial (b) and sagittal (c) FDG PET/CT showing increased FDG uptake around the screws and the adjacent muscles of the spine. Infection of the soft tissues and metallic device was confirmed intraoperatively and the metallic device was removed.

Figure 5. 72-year old man with suspicion for metallic implant associated infection of the right femur. The patient had a history of chronic osteomyelitis in the past due to an operated liposarcoma of the right thigh. Maximum-intensity-projection MIP (a) shows increased FDG uptake around the metallic implant in the right lateral femur (black arrow). Axial PET/CT demonstrates focally increased FDG along the metallic material and the adjacent femur (b, c, white arrows). Diagnosis of an infected metallic implant with osteomyelitis was established.

Figure 6. 57-year old man one year after implantation of a prosthetic ankle joint on the right side. Perfusion (a), blood pool (b) and osseous phase (c) of bone scintigraphy show markedly increased activity around the right ankle joint. Additionally $99 \mathrm{~m}$ Tc-labeled 
antigranulocyte antibody scintigraphy (d) was performed showing again increased uptake in the right ankle joint. Diagnosis of septic arthritis of the right prosthetic ankle joint was confirmed intraoperatively.

Figure 7. 48-year old woman with bacteriaemia and septic polyarthritis. Staphylococcus aureus was found in the left ankle joint. FDG PET/CT was performed for screening of involved joints. Maximum-intensity-projection MIP shows multiple joints (left shoulder, right sternoclavicular joint, left wrist, both knees and ankles, arrows) with increased FDG uptake. 